Original Research

\title{
Impact of subjective dizziness on motor and non-motor symptoms in patients with early stages of Parkinson's disease
}

\author{
Kyum-Yil Kwon ${ }^{1, *}$, Suyeon Park ${ }^{2}$, Eun Ji Lee ${ }^{1}$, Mina Lee ${ }^{1}$, Hyunjin Ju ${ }^{1}$ \\ ${ }^{1}$ Department of Neurology, Soonchunhyang University Seoul Hospital, Soonchunhyang University School of Medicine, 04401 Seoul, Republic of Korea \\ ${ }^{2}$ Department of Biostatistics and Data Innovation, Soonchunhyang University Seoul Hospital, Soonchunhyang University School of Medicine, 04401 Seoul, \\ Republic of Korea \\ *Correspondence: denovo78@naver.com (Kyum-Yil Kwon)
}

DOI:10.31083/j.jin2101003

This is an open access article under the CC BY 4.0 license (https://creativecommons.org/licenses/by/4.0/).

Submitted: 15 August 2021 Revised: 15 October 2021 Accepted: 31 October 2021 Published: 20 January 2022

Clinicians sometimes encounter patients with Parkinson's disease complaining of dizziness in real clinical settings. We sought to identify the relationship between self-perceived dizziness and motor or non-motor symptoms, especially in Parkinsonian patients in the early stages. Eight-six patients with less than five years of Parkinson's disease duration were recruited. We used the dizziness handicap inventory to access self-reported dizziness in patients with early Parkinson's disease. Parkinsonian motor symptoms such as postural instability and gait difficulty and non-motor features for global cognitive function, depressive mood, anxiety state, fatigue state, and autonomic dysfunction were measured using representative scales. Linear regression analysis demonstrated that the dizziness handicap inventory score was significantly related to postural instability and gait difficulty, anxiety, gastrointestinal, and cardiovascular domain of dysautonomia. In addition, the dizziness handicap inventory score was positively correlated with scores for postural instability and gait difficulty, anxiety, gastrointestinal, and cardiovascular dysautonomia. We found that self-reported dizziness was highly linked to postural instability and gait difficulty, anxiety, gastrointestinal and cardiovascular dysfunctions in patients with early Parkinson's disease. Further follow-up studies on the association between dizziness and the pathophysiology of Parkinson's disease are needed.

Keywords

Dizziness; Dysatonomia; Motor; Non-motor; Parkinson's disease

\section{Introduction}

Dizziness could manifest as different types of vertigo, faintness, or light-headedness. Moreover, a recent study showed that dizziness in patients with early Parkinson's disease (PD) might be of various types, although orthostatic dizziness (about 40.5\%) was the most common type of dizziness [1].

Between $46 \%$ and $68 \%$ of patients with PD complain of subjective dizziness [1-4]. Some have supposed that dizziness might be caused by orthostatic hypotension $[5,6]$, whereas others have asserted that dizziness itself is not indicative of orthostatic hypotension in patients with PD [1, 4]. Also, patients with preclinical or de novo PD could occasionally present with dizziness $[4,7]$. potentially suggesting that dizziness is connected with the pathophysiology of the disease. However, the clinical implications of patients with PD complaining about dizziness have remained little known until now.

Dizziness may significantly affect the quality of life or decrease the functional activity of individuals. Among various types of dizziness-related questionnaires to measure subjective dizziness, the Dizziness Handicap Inventory (DHI) has been most widely used to evaluate the effect of self-perceived dizziness on daily life $[8,9]$. Furthermore, the DHI has recently been applied to access dizziness-related disability or quality of life in patients with PD [2,3]. We sought to investigate the detailed association between the DHI and clinical characteristics of patients with early stages of PD.

\section{Materials and methods}

\subsection{Patients}

The ethics committee approved this retrospective study with a waiver of individual informed consent of our hospital (IRB No. 2020-03-023). The PD registry of our movement disorders clinics between July 2017 and March 2020 and their patient medical records was consecutively analyzed. The clinical diagnosis of PD was made according to the diagnostic criteria of UK Brain Bank [10]. All patients performed brain magnetic resonance imaging (MRI) and dopamine transporter (DAT) scanning.

Patients with PD with early stages of the disease (less than 5 years of its duration) were selected More strict exclusion criteria were applied to increase the diagnostic precision of PD. Subjects did not reveal a characteristic pattern of rostrocaudal gradient in the DAT findings [11] or showed significant alleviation of motor symptoms clinically despite proper antiparkinsonian medications during follow-up. Subjects with significant abnormalities, including significant vascular burden or ventricular enlargement on brain MRI, were excluded [12]. Furthermore, any individuals with atypical features that could imply the possibility of atypical parkinsonism were excluded. 
Patients with vestibular disorders, serious medical problems, including uncontrolled diabetes mellitus, cancer/paraneoplastic syndrome, clinically relevant cardiac problem, liver cirrhosis, and severe renal failure, or any structural lesions in the brainstem or cerebellum were also excluded, as those factors might cause significant dizziness irrelevant to PD itself [13].

Of a total of 104 patients with early PD, 86 were finally included in the study after excluding 18 patients (Specifically, four patients had multiple system atrophy, 1 had progressive supranuclear palsy, 3 had normal pressure hydrocephalus, 3 had vascular parkinsonism, 1 had essential tremor, 2 had drug-induced parkinsonism, 1 had dementia with Lewy bodies, and 3 had unspecified parkinsonism).

\subsection{Clinical assessments}

At the time of registration in our movement disorders clinic, we have routinely investigated clinical details of demographics and various symptoms in PD. For the evaluation of motor symptoms, the Unified Parkinson's Disease Rating Scale (UPDRS) part III and the motor subscores for tremor, rigidity, bradykinesia, and postural instability/gait difficulty (PIGD), in addition to Hoehn and Yahr (HY) stage have been evaluated as previously described $[13,14]$. For the evaluation of various non-motor symptoms (NMSs), we have evaluated the Korean version of the Montreal Cognitive Assessment (MoCA-K) for global cognitive status [15], the Korean version of the Beck Depression Inventory (BDI) for depression [16], the Korean version of the Beck Anxiety Inventory (BAI) for anxiety state [17], the Parkinson's Disease Fatigue Scale (PFS) for fatigue symptom [18, 19], and the Korean version of the Scale for Outcomes in Parkinson's Disease-Autonomic (SCOPA-AUT) for autonomic dysfunction [20].

Furthermore, we routinely assessed self-reported dizziness using the Korean version of the DHI $[3,21]$. The DHI consists of 25 items (score of 0,2 , or 4 for each item), and its scores range from 0 to 100; a higher score represents a more severe degree of subjective dizziness.

\subsection{Statistics}

We used descriptive statistics to analyze the data and univariable and multivariable linear regression analyses to find the association of the DHI score with clinical characteristics of the motor and non-motor symptoms. We performed partial correlation analysis between the DHI score and clinical characteristics after adjusting for age, gender, and disease duration. We defined a $p<0.05$ as statistically significant. We did all statistical analyses using SPSS (version 20.0, IBM Corp., Armonk, NY, USA) and Rex version 3.6.3 (RexSoft Inc., Seoul, Korea; URL http://rexsoft.org).

\section{Results \\ 3.1 Demographics and clinical characteristics in patients with early $P D$}

Demographic and clinical findings of patients with early $\mathrm{PD}$ are described in Table 1 . In our study population, the age
Table 1. Demographics and clinical characteristics in 86 patients with early Parkinson's disease (PD).

\begin{tabular}{|c|c|}
\hline & Patients with early PD $(n=86)$ \\
\hline Age, year & $71.1 \pm 8.5$ \\
\hline Gender-female & $49(57.0 \%)$ \\
\hline Disease duration at registration, year & $1.9 \pm 1.4$ \\
\hline Follow-up duration, year & $2.8 \pm 0.6$ \\
\hline Body mass index, $\mathrm{kg} / \mathrm{m}^{2}$ & $23.6 \pm 3.3$ \\
\hline Level of education, year & $9.2 \pm 4.9$ \\
\hline Levodopa equivalent daily dose, $\mathrm{mg}$ & $139.4 \pm 218.6$ \\
\hline \multicolumn{2}{|l|}{ UPDRS-III } \\
\hline Tremor score & $2.7 \pm 2.2$ \\
\hline Rigidity score & $4.7 \pm 2.7$ \\
\hline Bradykinesia score & $10.9 \pm 4.6$ \\
\hline Postural instability-gait disorder score & $2.9 \pm 2.7$ \\
\hline Total motor score & $23.3 \pm 8.9$ \\
\hline Hoehn and Yahr stage & $2.2 \pm 0.4$ \\
\hline Montreal Cognitive Assessment & $21.3 \pm 5.3$ \\
\hline Beck depression inventory & $10.2 \pm 7.2$ \\
\hline Beck anxiety inventory & $5.9 \pm 6.1$ \\
\hline Parkinson's disease fatigue scale & $40.6 \pm 15.7$ \\
\hline \multicolumn{2}{|l|}{ SCOPA-AUT (dysautonomia) } \\
\hline Gastrointestinal (GI) domain & $4.2 \pm 3.3$ \\
\hline Urinary (UR) domain & $7.0 \pm 4.5$ \\
\hline Cardiovascular (CV) domain & $1.0 \pm 1.6$ \\
\hline Thermoregulatory (TR) domain & $0.7 \pm 1.2$ \\
\hline Pupillomotor (PM) domain & $0.4 \pm 1.1$ \\
\hline Sexual (SX) domain & $3.8 \pm 2.5$ \\
\hline Total score & $14.2 \pm 8.2$ \\
\hline Dizziness Handicap Inventory & $7.7 \pm 13.5$ \\
\hline
\end{tabular}

Data are represented as $n$ (\%) or mean \pm S.D. values. PD, Parkinson's disease; UPDRS, Unified Parkinson's disease rating scale; SCOPAAUT, Scales for Outcomes in Parkinson's Disease-Autonomic questionnaire.

of the 86 patients was $71.1 \pm 8.5$ (mean \pm S.D) years, and 49 (57.0\%) of the patients were women. The disease duration was $1.9 \pm 1.4$ years. The detailed scores of motor symptoms were as follows: $23.3 \pm 8.9$ for the total motor score, $2.7 \pm$ 2.2 for tremor score, $4.7 \pm 2.7$ for rigidity score, $10.9 \pm 4.6$ for bradykinesia score, $2.9 \pm 2.7$ for PIGD score. The HY stage was $2.2 \pm 0.4$. Each score of NMSs was as follows: 21.3 \pm 5.3 for MoCA-K, $10.2 \pm 7.2$ for BDI, $5.9 \pm 6.1$ for BAI, $40.6 \pm 15.7$ for PFS, $14.2 \pm 8.2$ for SCOPA-AUT. The DHI score was $7.7 \pm 13$.

In this study, 32 patients with PD took anti-dopaminergic drugs (16 were taking both levodopa and dopamine agonist, 11 were taking levodopa, and 5 were taking dopaminergic agonist). In comparison, 54 patients with PD were drug naïve de novo. There was no difference in the DHI score between de novo patients and the others; the DHI score of 32 patients with PD taking drugs was $7.4 \pm 13.7$, and the DHI score of 54 patients with de novo drug naïve of $\mathrm{PD}$ was $7.8 \pm 13.5$ $(p$-value $=0.911)$. 
(A)
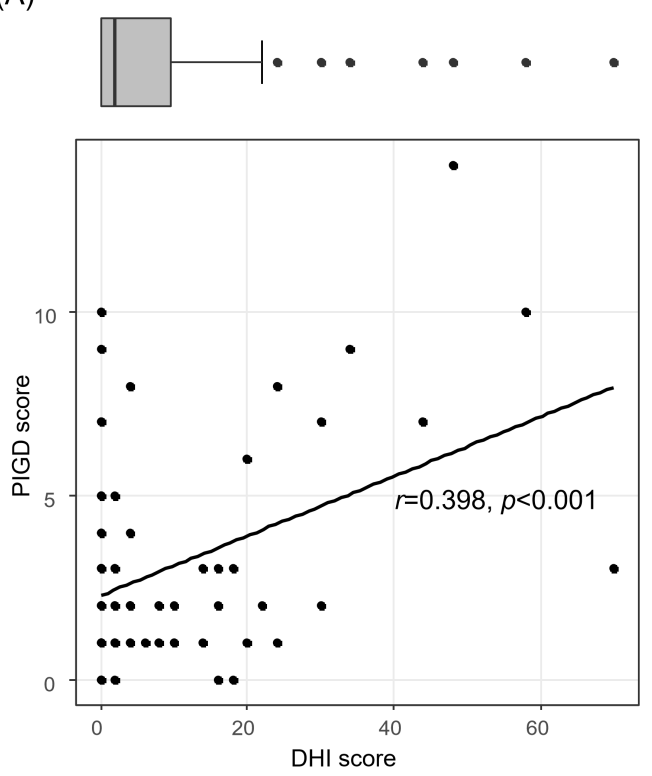

(C)
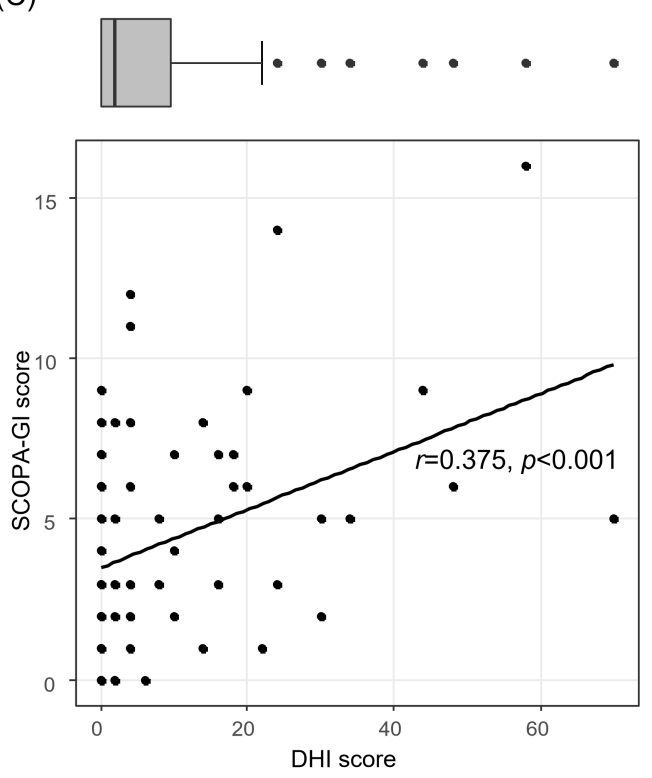

(B)
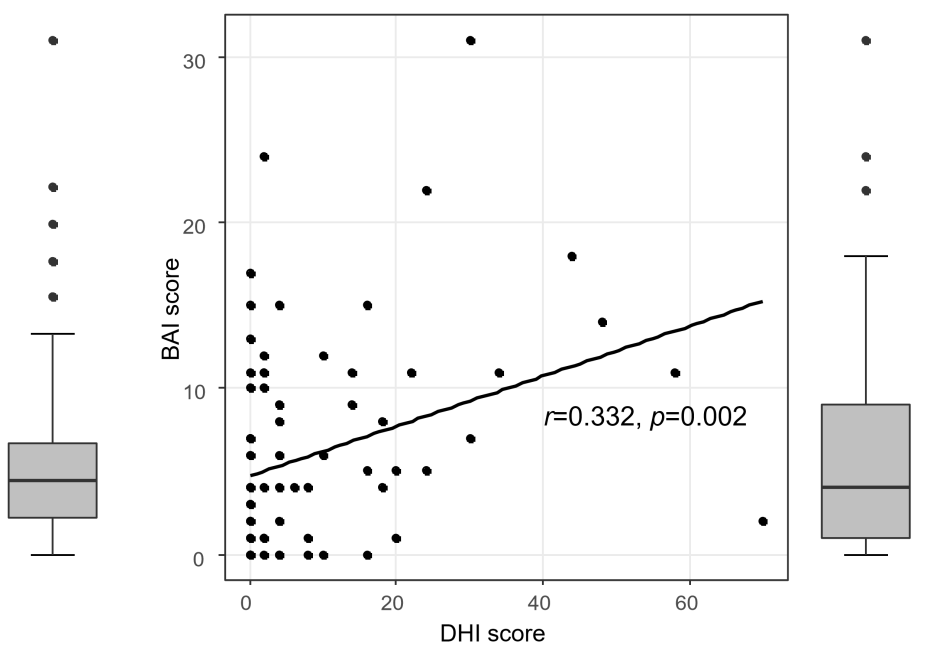

(D)
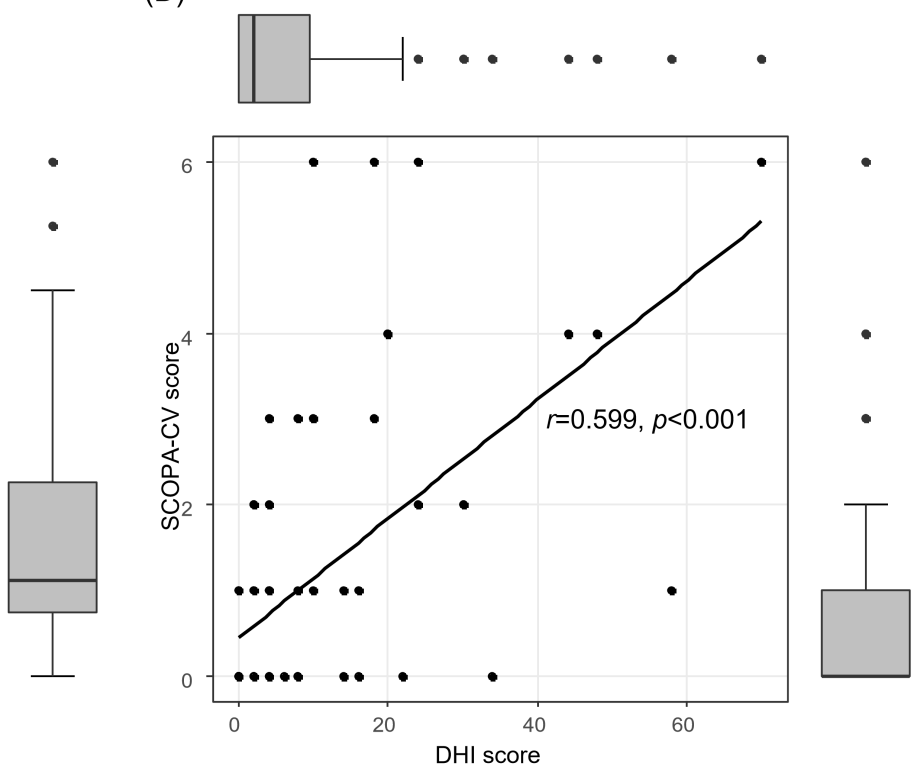

Fig. 1. Scatter plots showing partial correlations between Dizziness Handicap Inventory (DHI) scores and significant clinical characteristics in patients with early Parkinson's disease after adjusting for age, gender, and disease duration. (A) The DHI score was correlated with the postural instability gait disorder (PIGD) score $(r=0.398, p<0.001)$. (B) The DHI score was associated with the Beck Anxiety Inventory (BAI) score $(r=0.332, p=$ 0.002). (C) The DHI score was associated with the gastrointestinal (GI) domain score of autonomic dysfunction $(r=0.375, p<0.001)$. (D) The DHI score was linked to the cardiovascular (CV) domain score of autonomic dysfunction $(r=0.599, p<0.001)$.

\subsection{Linear regression analyses of subjective dizziness in patients with early $P D$}

In the left panel of Supplementary Table 1, univariable linear regression analyses showed that the DHI score was significantly related to the scores for PIGD, MoCA-K, BDI, BAI, PFS, a total score of SCOPA-AUT, and the gastrointestinal (GI) domain and cardiovascular (CV) domain of SCOPAAUT. Moreover, in the right panel of Supplementary Table 1, multivariable linear regression analysis revealed that the DHI score was associated with scores for PIGD $(\beta=1.304$, $p=0.005)$, BAI $(\beta=0.466, p=0.013)$, GI domain of dysautonomia $(\beta=0.873, p=0.039)$, and $\mathrm{CV}$ domain of dysautono$\operatorname{mia}(\beta=4.856, p<0.001)$.

\subsection{Correlations between the DHI and significant clinical factors in patients with early $P D$}

As a post hoc analysis, we conducted partial correlation analyses between the DHI score and the significant clinical characteristics derived from the linear regression analyses (Supplementary Table 1). After adjusting for age, gender, and disease duration, the DHI score is positively correlated 
with the scores of PIGD $(r=0.398, p<0.001)$, BAI $(r=0.332$, $p=0.002)$, GI domain of SCOPA-AUT $(r=0.375, p<0.001)$, and CV domain of SCOPA-AUT $(r=0.599, p<0.001)$, as shown in Fig. 1.

\section{Discussion}

We did not investigate the etiologies of dizziness in participants in this study. Instead, we assessed the severity of subjective dizziness using the DHI, a representative scale for quantifying self-reported dizziness, to investigate the association with motor or non-motor symptoms, regardless of the types of dizziness. We found that a complaint of dizziness in patients with early PD was significantly associated with some factors of motor and non-motor symptoms.

From the beginning of the study design, we assumed that the early stages of PD may appropriately reflect the diversity of dizziness, including any potential pathophysiology. In general, some antiparkinsonian medications may induce dizziness; moreover, medication-related orthostatic hypotension could commonly occur in patients with relatively advanced stages of PD. Therefore, we enrolled Parkinsonian patients only with the early stages of the disease to recruit a relatively homogeneous population of PD and weaken medication-related adverse effects. As a result, unexpectedly, we found that dizziness was linked to PIGD, anxiety, and autonomic dysfunction in patients with early PD. Based on this, it is reasonable to infer that a complaint of dizziness by patients with early PD might be implicated in the pathophysiology of the disease.

In line with our results, dizziness has been reported in patients with preclinical or de novo stages of PD [7,22]. Notably, we found that subjective dizziness was related to the PIGD motor symptom in patients with early PD. Until now, little was known about the association between dizziness and motor symptoms in patients with PD. Allcock et al. [23] showed that orthostatic hypotension was more frequently observed in patients with the PIGD phenotype of PD. We found that $\mathrm{CV}$ dysautonomia was significantly linked to subjective dizziness in patients with early PD. Others demonstrated that CV dysautonomia might be connected with a subclinical status of orthostatic hypotension-related dizziness [6, 24]. Moreover, the non-tremor dominant subtype, including the PIGD phenotype, is associated with autonomic dysfunctions, including orthostatic hypotension [25]. Our findings suggest that some pathophysiologic connections between dizziness, PIGD motor symptom, and CV dysautonomia, including orthostatic hypotension, might exist.

Anxiety and depression are common and could overlap in patients with PD. However, their neuropsychiatric symptoms showed distinct features, and their pathophysiology remains to be further elucidated. A review article showed that anxiety is related to dopaminergic depletion in the caudate nucleus and the putamen. In contrast, depression is involved in the functional connectivity of the prefrontal-limbic networks [26]. In patients with early PD, we demonstrated that dizziness was associated with anxiety but not with depression. Therefore, our findings suggest that dizziness's pathogenesis is implicated in the striatonigral dopaminergic pathway rather than in the prefrontal-limbic networks. However, a well-designed study with larger samples is warranted to address this issue.

We preferred to use the SCOPA-AUT among several questionnaires to evaluate autonomic dysfunctions since a previous review recommended that the SCOPA-AUT be used as one of the dysautonomia rating scales [27]. The SCOPA-AUT consists of several items for each autonomic domain, thereby enabling grading of the severity of each domain of dysautonomia [20]. We found specifically that the GI and CV domains among several autonomic dysfunctions were significantly linked to subjective dizziness in early PD. Our results strongly suggest that dizziness is highly associated with certain types of dysautonomia in patients with early PD. The CV domain questionnaires are mainly focused on orthostatic dizziness or syncope, indicating orthostatic hypotension.

In our study population, only 63 patients with early PD were evaluated for orthostatic hypotension by active blood pressure monitoring, resulting in 14 subjects with orthostatic hypotension. Orthostatic hypotension showed a tendency to a higher DHI score, inferring that subjective dizziness might be in part associated with orthostatic dizziness. Intriguingly, we first discovered that the GI domain was significantly connected with subjective dizziness in patients with early PD. Exactly why dizziness in patients with early PD is correlated with GI dysautonomia remains unknown, but perhaps the pathophysiologic mechanism of Lewi body pathology ascending from the gut might be linked to that of dizziness in patients with PD. However, the detailed relationship between dizziness and GI dysautonomia in patients with PD remains to be further elucidated.

The present study has several limitations. First, selection bias might exist for a retrospective design, although we have evaluated patients presenting with parkinsonism according to a constant protocol. Second, we did not do detailed tests to differentiate the types of dizziness in the participants with PD. However, our purpose was not focused on the relationship between clinical features and different types of dizziness etiology. We supposed that our assessments might be sufficient to meet our primary goal, as described previously. Third, our findings should be cautiously generalized to all populations with $\mathrm{PD}$, as we investigated only patients with early PD in this study, implying that we could not completely exclude the possibility of atypical parkinsonism due to the lack of long-term follow-ups. Fourth, our findings should be cautiously generalized to all populations with PD since we investigated only patients with early PD.

\section{Conclusions}

In conclusion, we found that a complaint of dizziness in patients with early PD might suggest more severe symptoms 
for PIGD, anxiety, GI dysautonomia, and CV dysautonomia. More well-designed studies with more patients with PD are needed to confirm our results.

\section{Abbreviations}

PD, Parkinson's disease; DHI, Dizziness Handicap Inventory; MRI, magnetic resonance imaging; DAT, dopamine transporter; UPDRS, Unified Parkinson's Disease Rating Scale; PIGD, postural instability and gait disorder; NMS, non-motor symptom; MoCA-K, Korean version of the Montreal Cognitive Assessment; BDI, Beck Depression Inventory; BAI, Beck Anxiety Inventory; PFD, Parkinson's Disease Fatigue Scale; SCOPA-AUT, Scale for Outcomes in Parkinson's Disease-Autonomic; GI, gastrointestinal; CV, cardiovascular.

\section{Author contributions}

KYK-Conceptualization, methodology, data curation, formal analysis, funding acquisition, investigation, writing and original draft, writing-review\&editing and supervision. SP-Methodology, formal analysis, writingreview\&editing. EJL-Data curation, investigation and writing-review\&editing. ML-Data duration, investigation and writing-review\&editing. HJ-Data duration, investigation and writing-review\&editing. All authors have read and agreed to the published version of the manuscript.

\section{Ethics approval and consent to participate}

All procedures were performed in accordance with ethical standards of the institution and/or the national research committee as well as with the 1964 Helsinki Declaration and its subsequent amendments. This study was retrospective and was approved with waiver of individual informed consent by the ethics committee of our Institutional Review Board (IRB No. 2020-03-023).

\section{Acknowledgment}

We thank all staffs in our department of Neurology for their kind and enthusiastic support.

\section{Funding}

This study was supported by the Soonchunhyang University Research Fund (Grant No. 20200410) and the Korea government (the Ministry of Science and ICT, the Ministry of Trade, Industry and Energy, the Ministry of Health \& Welfare, Republic of Korea, the Ministry of Food and Drug Safety) (No. KMDF202013C13-03).

\section{Conflict of in terest}

The authors declare no conflict of interest.

\section{Supplementary material}

Supplementary material associated with this article can be found, in the online version, at https://www.imrpress.com/j ournal/JIN/21/1/10.31083/j.jin2101003.

\section{References}

[1] Yilmaz L, Tunç T, İnan LE. The Causes of Dizziness in Parkinson's Disease. International Journal of Clinical Medicine. 2014; 05: 667673.

[2] van Wensen E, van Leeuwen RB, van der Zaag-Loonen HJ, Masius-Olthof S, Bloem BR. Benign paroxysmal positional vertigo in Parkinson's disease. Parkinsonism \& Related Disorders. 2013; 19: 1110-1112.

[3] Lee DY, Yang HJ, Yang DS, Choi JH, Park BS, Park JY. Rasch analysis of the clinimetric properties of the Korean dizziness handicap inventory in patients with Parkinson's disease. Research in Vestibular Science. 2018;17: 152-159.

[4] Kwon K, Park S, Lee M, Ju H, Im K, Joo B, et al. Dizziness in patients with early stages of Parkinson's disease: Prevalence, clinical characteristics and implications. Geriatrics \& Gerontology International. 2020; 20: 443-447.

[5] Hillen ME, Wagner ML, Sage JI. "Subclinical" orthostatic hypotension is associated with dizziness in elderly patients with Parkinson's disease. Archives of Physical Medicine and Rehabilitation. 1996; 77: 710-712.

[6] Goldstein DS, Holmes CS, Dendi R, Bruce SR, Li S. Orthostatic hypotension from sympathetic denervation in Parkinson's disease. Neurology. 2002; 58: 1247-1255.

[7] Schrag A, Horsfall L, Walters K, Noyce A, Petersen I. Prediagnostic presentations of Parkinson's disease in primary care: a casecontrol study. The Lancet. Neurology. 2015; 14: 57-647

[8] Jacobson GP, Newman CW. The development of the Dizziness Handicap Inventory. Archives of Otolaryngology $\urcorner$-head and Neck Surgery. 1990; 116: 424-427.

[9] Mutlu B, Serbetcioglu B. Discussion of the dizziness handicap inventory. Journal of Vestibular Research. 2013; 23: 271-277.

[10] Gelb DJ, Oliver E, Gilman S. Diagnostic criteria for Parkinson's disease. Archives of neurology. 1999; 56: 33-39.

[11] Oh M, Kim JS, Kim JY, Shin KH, Park SH, Kim HO, et al. Subregional patterns of preferential striatal dopamine transporter loss differ in Parkinson disease, progressive supranuclear palsy, and multiple-system atrophy. Journal of Nuclear Medicine. 2012; 53: 399-406.

[12] Heim B, Krismer F, De Marzi R, Seppi K. Magnetic resonance imaging for the diagnosis of Parkinson's disease. Journal of Neural Transmission (Vienna, Austria: 1996). 2017; 124: 915-964.

[13] Jankovic J, McDermott M, Carter J, Gauthier S, Goetz C, Golbe L, et al. Variable expression of Parkinson's disease: a base-line analysis of the DATATOP cohort. the Parkinson Study Group. Neurology. 1990; 40: 1529-1534.

[14] Kwon KY, Park S, Lee HM, Park YM, Kim J, Kim J, et al. Backward Gait is Associated with Motor Symptoms and Fear of Falling in Patients with De Novo Parkinson's Disease. Journal of Clinical Neurology. 2019; 15: 473-479.

[15] Lee J, Lee DW, Cho S, Na DL, Jeon HJ, Kim S, et al. Brief screening for mild cognitive impairment in elderly outpatient clinic: validation of the Korean version of the Montreal Cognitive Assessment. Journal of Geriatric Psychiatry and Neurology. 2008; 21: 104-110.

[16] Jo SA, Park MH, Jo I, Ryu S, Han C. Usefulness of Beck Depression Inventory $(\mathrm{BDI})$ in the Korean elderly population. International Journal of Geriatric Psychiatry. 2007; 22: 218-223.

[17] Lee K, Kim D, Cho Y. Exploratory Factor Analysis of the Beck Anxiety Inventory and the Beck Depression Inventory-II in a Psychiatric Outpatient Population. Journal of Korean Medical Science. 2018; 33: e128.

[18] Brown RG, Dittner A, Findley L, Wessely SC. The Parkinson fatigue scale. Parkinsonism \& Related Disorders. 2005; 11: 49-55.

[19] Ahn S, Lee J, Chu SH, Sohn YH. Uncertainty and depression in people with Parkinson's disease: a cross-sectional study. Nursing \& Health Sciences. 2017; 19: 220-227.

[20] Kim J, Song I, Koh S, Ahn T, Kim SJ, Cheon S, et al. Validation of the Korean Version of the Scale for Outcomes in Parkinson's Disease-Autonomic. Journal of Movement Disorders. 2017; 10: 
29-34.

[21] Han GC, Lee EJ, Lee JH, Park SN, Lee HY, Jeon EJ, et al. The study of standardization for a Korean adaptation of self-report measures of dizziness. Journal of the Korean Balance Society. 2004; 3: 307325.

[22] Barone P, Antonini A, Colosimo C, Marconi R, Morgante L, Avarello TP, et al. The PRIAMO study: a multicenter assessment of nonmotor symptoms and their impact on quality of life in Parkinson's disease. Movement Disorders. 2009; 24: 1641-1649.

[23] Allcock LM, Kenny RA, Burn DJ. Clinical phenotype of subjects with Parkinson's disease and orthostatic hypotension: autonomic symptom and demographic comparison. Movement Disorders. 2006; 21: 1851-1855.

[24] Park J, Kim H, Park KM, Ha SY, Kim SE, Shin KJ, et al. Ortho- static dizziness in Parkinson's disease is attributed to cerebral hypoperfusion: a transcranial doppler study. Journal of Clinical Ultrasound. 2017; 45: 337-342.

[25] Marras C, Chaudhuri KR. Nonmotor features of Parkinson's disease subtypes. Movement Disorders. 2016; 31: 1095-1102.

[26] Wen M, Chan LL, Tan LCS, Tan EK. Depression, anxiety, and apathy in Parkinson's disease: insights from neuroimaging studies. European Journal of Neurology. 2016; 23: 1001-1019.

[27] Pavy-Le Traon A, Amarenco G, Duerr S, Kaufmann H, Lahrmann $\mathrm{H}$, Shaftman SR, et al. The Movement Disorders task force review of dysautonomia rating scales in Parkinson's disease with regard to symptoms of orthostatic hypotension. Movement Disorders. 2011; 26: 1985-1992. 